\title{
Deep Convolutional Neural Networks for Accurate Diagnosis of COVID-19 Patients Using Chest X-Ray Image Databases from Italy, Canada, and the USA
}

\author{
Amgad A. Salama ${ }^{1}$, PhD; Samy H. Darwish², PhD; Samir M. Abdel-Mageed ${ }^{3}$, PhD; Radwa A. Meshref ${ }^{4}$, PhD; Ehab I. Mohamed ${ }^{5 *}$, PhD DSc \\ ${ }^{1}$ Research and Development Center, Air Defense College, Alexandria, Egypt; ${ }^{2}$ Electrical Engineering Department, Faculty of Engineering, Pharos University, Alexan- \\ dria, Egypt; ${ }^{3}$ Physics Department, Faculty of Science, Alexandria University, Alexandria, Egypt; ${ }^{4}$ Medical Equipment Department, Faculty of Allied Medical Sciences, \\ Pharos University, Alexandria, Egypt; ${ }^{5}$ Medical Biophysics Department, Medical Research Institute, Alexandria University, Alexandria, Egypt
}

*eimohamed@yahoo.com; ehab.abdo@alexu.edu.eg

Recommended Citation: Salama AA, Darwish SH, Abdel-Mageed SM, Meshref RA, Mohamed El. Deep convolutional neural network for accurate diagnosis of COVID-19 patients using chest X-ray image database from Italy, Canada, and the USA. Univ Louisville J Respir Infect 2021; 5(1):Article 34. doi: 10.18297/jri/vol5/iss1/34.

\begin{abstract}
Introduction: Severe acute respiratory syndrome coronavirus 2 (SARS-CoV-2), famously known as COVID-19, has quickly become a global pandemic. Chest X-ray (CXR) imaging has proven reliable, fast, and cost-effective for identifying COVID-19 infections, which presents with atypical unilateral patchy infiltration in the lungs like typical pneumonia. We employed the deep convolutional neural network (DCNN) ResNet-34 to detect and classify CXR images from patients with COVID-19, other viral pneumonias, and normal controls.
\end{abstract}

Methods: We created a single database, containing 781 source CXR images for COVID-19 $(n=240)$, other viral pneumonias $(n=274)$, and normal controls $(n=267)$ from four different international sub-databases: the Società Italiana di Radiologia Medica e Interventistica (SIRM), the GitHub Database, the Radiology Society of North America (RSNA), and the Kaggle Chest X-Ray Database. Images were resized, normalized without any augmentation, and arranged in

\section{Introduction}

Since December 2019, the severe acute respiratory syndrome coronavirus 2 (SARS-CoV-2), famously known as COVID-19, has quickly become a global pandemic. In fact, as of October 14, 2021, reports to the World Health Organization (WHO) have amounted to $239,007,759$ confirmed cases, including $4,871,841$ deaths.[1] The pandemic continues to have disastrous effects on health, industry, and social life worldwide. The disease's long-term symptoms range from flu-like signs to severe respiratory distress syndrome. Patients exhibit evident anomalies (i.e., opacities and lung consolidations) in chest X-ray (CXR) images.[2, 3] Rapid $m$ batches of 16 images before supervised training, testing, and cross-validation of the DCNN classifier.

Results: The ResNet-34 had a diagnostic accuracy as of the receiver operating characteristic (ROC) curves of the truepositive rate versus the false-positive rate with the area under the curve (AUC) of 1.00, 0.99, and 0.99, for COVID-19, other viral pneumonia, and normal control CXR images, respectively. This accuracy implied identical high sensitivity and specificity values of $100 \%, 99 \%$, and $99 \%$ for the three groups, respectively. ResNet-34 achieved identical sensitivity and specificity of $100 \%, 99.6 \%$, and $98.9 \%$ for classifying CXR images of the three groups, with an overall accuracy of $99.5 \%$ for the testing subset for diagnosis/prognosis.

Conclusion: Based on this high classification precision, we believe that the output activation map of the final layer of the ResNet-34 is a powerful tool for the accurate diagnosis of COVID-19 infection from CXR images. and accurate detection of COVID-19 is crucial to control outbreaks in the community and hospitals.[4] Reverse transcriptase quantitative polymerase chain reaction (RT-qPCR) testing, which can detect SARS-CoV2 RNA in different types of clinical specimens, is the primary technique used for diagnosis. Nevertheless, RT-qPCR testing is time-consuming, complex, and its sensitivity is highly variable with a high probability of false detection. $[2,5]$

Medical imaging techniques such as CXR and computed tomography (CT) have proven reliable, fast, and cost-effective for detecting lung diseases and identifying COVID-19 infections compared to RT-qPCR.[6] Al- 
Table 1. Examples of machine learning and artificial intelligence techniques in different medical fields

\begin{tabular}{cllll}
\hline Reference & Domain & Technique & Technology & Topic \\
\hline$[10]$ & Radiology & Ultrasound & Regression & Breast cancer \\
{$[11]$} & Radiology & CT & $\mathrm{ML}$ & Lungs \\
{$[12]$} & Radiology & $\mathrm{MRI}$ & $\mathrm{ML}$ & Brain \\
{$[13]$} & Radiology & X-ray & $\mathrm{ML}$ & Chest \\
{$[14]$} & Hematology & Laboratory & $\mathrm{ML}$ & Biological hematology \\
{$[15]$} & Hematology & HSCT & $\mathrm{ML}$ & aGVHD \\
{$[16]$} & Hematology & Flow cytometry & $\mathrm{Al}$ & Leukemia \\
{$[17]$} & Hematology & Flow cytometry & $\mathrm{Al}$ & Leukemia \\
{$[18]$} & Hematology & Cytometry & $\mathrm{Al}$ & AML \\
{$[19]$} & Hematology & Laboratory & $\mathrm{DM}$ & $\mathrm{PV}$ \\
\hline
\end{tabular}

Abbreviations: aGVHD, acute graft-versus-host disease; $\mathrm{Al}$, artificial intelligence; AML, acute myeloid leukemia; CT, computed tomography; DM, data mining; HSCT, hematopoietic stem cell transplantation; MRI, magnetic resonance imaging; PV, Polychthemia Vera.

though CXR images do not show any irregularities at the beginning of infection, as COVID-19 proceeds, it slowly presents with atypical unilateral patchy infiltration in the lungs, which exhibit similarities to the characteristics of other pneumonias.[7] Computer vision algorithms have shown high performance in object detection, image classification, and indexing for retrieval and annotation at a large scale.[3] Of these, deep convolutional neural networks (DCNN) with transfer learning have distinguished themselves in many computervision tasks, such as image classification, especially in the field of radiology for computer-aided diagnosis of chronic lung diseases from CT Hounsfield unit values or texture features.[3, 8, 9]

Transfer learning is a supervised machine learning technique, which reuses parts of a previously trained model in a specific field for another task in a new model, with the advantages of using less training data, better generalization, and affordable deep learning.[1015] Examples of the machine learning and artificial intelligence techniques already being tested or applied in various medical fields are summarized in Table 1.[1019] Applying transfer learning to a pre-trained residual neural network (ResNet) has been found very useful as a starting point for training a new DCNN for image recognition by modifying the activation function Softmax and the classification layer. Using the pre-trained weights accelerates the training phase, employing only the available small medical datasets, which avoids losing a tremendous amount of time for training by large datasets if one develops a new DCNN from scratch.[20]

The objective of the present study was to employ the DCNN ResNet-34 for the detection and classification (diagnosis/prognosis) of CXR images from patients with COVID-19, other viral pneumonias, and normal controls.[21]

\section{Methods}

Study population database and sample size

We calculated the sample size using STATA statistical software version 9 (StataCorp LLC, College Station, TX, USA). The diagnosis of COVID-19 by the RT-qPCR technique was considered the gold standard. We aimed to achieve a confidence level of $99 \%$ and a margin of error of $1 \%$ for the proposed DCNN model; hence, the sample size should include at least 722 CXR images. Thus, we created a single database containing 781 source CXR images for COVID-19 $(n=240)$, other viral pneumonias $(n=274)$, and normal controls $(n=267)$ from four different international sub-databases to train, validate, and test the DCNN diagnosis/prognosis model. These four databases were the following: the Società Italiana di Radiologia Medica e Interventistica (SIRM) COVID-19 Database, reporting 384 COVID-19 positive CXR images [22]; the GitHub Database by Cohen et al., containing 319 CXR images of COVID-19, severe acute respiratory syndrome, Middle East respiratory syndrome, and acute respiratory distress syndrome [23]; the Radiology Society of North America (RSNA) Pneumonia-Detection-Challenge Database, comprising 108,948 frontal-view CXR images of 32,717 unique patients for classification and localization of common thorax diseases using a DCNN [24]; and finally, the Kaggle Chest X-Ray Database, containing 5,247 CXR images of normal controls and viral and bacterial pneumonia patients.[25]

\section{Deep convolutional neural network model selection}

A supervised pre-trained ResNet-34, a benchmarked DCNN model with an error rate as low as $3.6 \%$, was trained, validated, and tested to detect, identify, and classify our database of 781 CXR images.[21] We trained the multilayer feed-forward ResNet-34 model with backpropagation of errors using the Adam op- 
timization algorithm, with a learning rate of $\alpha=10^{-3}$ and a batch size of 16 random CXR images (i.e., of COVID-19, other viral pneumonias, or normal controls). The ResNet-34 structure has a moderate computational complexity, with 26.70 and 8.58 top- 1 and top-5 errors, respectively.[26] Then, we evaluated the model using the PyTorch Library of the Anaconda Package (2021 Anaconda Inc., New York, USA) running on a MacBook Pro (Intel i5-core @ 2.7 GHz processor and 16 GB RAM) under Mojave 10.14.6.[26]

Before applying the database of random inputs, CXR images were all pre-processed-i.e., resized to $224 \times$ 224 to match input requirements and normalized, without any image augmentation, according to standards of the ResNet-34-and were arranged in $m$ batches of 16 images. That is, we randomly divided the 781 CXR images into 49 batches of 16 images each; we carried out training out using 29 image batches $(60 \%)$, validation using ten batches $(20 \%)$, and testing for diagnosis using the remaining ten batches $(20 \%)$. As we passed CXR image batches through the ResNet-34 for training, batch normalization in between skips double- or triple-layers containing nonlinearities for image classification.[20]

To localize and highlight a critical region of interest (ROI) for a particular category within an input CXR image, we employed the Gradient-Weighted Class Activation Mapping (Grad-CAM) method to explain the DCNN prediction visually. It uses the gradient information of a target class flowing back into the last convolutional layer to generate visual explanations from the DCNN model. It generates a class activation map from a residual block of the DCNN model (Figures 1A and $\mathbf{1 B}$ ), where it feeds the global average pooled convolutional feature maps into the fully connected final output layer, which is defined as follows:[20]

$$
y=F^{k}\left(x, w_{k}^{c}\right)+x
$$

(Eq. 1)

where $x$ and $y$ are the input and output vectors of the building block, $F^{k}\left(x, w_{k}^{c}\right)$ is the residual mapping, $F^{k} \in \mathbb{R}^{u \times v}$ represents the global average pooling that spatially averages the $k$ th feature map of width $u$ and height $v$ produced from the last convolution layer of the DCNN, and $w_{k}^{c}$ is the weight connecting the $k$ th feature map to an output node corresponding to class $c$. For the residual block considered in Figure 1B, $F$ is given by:

$$
F=w_{2} \operatorname{ReLU}\left(w_{1} x\right)+x
$$

(Eq. 2)

where $\operatorname{ReLU}($.$) is the rectified linear unit and is given$ by:

$$
\operatorname{ReLU}(x)=\max (0, x)
$$

The prediction score (i.e., the input to the activation function Softmax) at the output layer, $S_{c}$, can be expressed as a weighted sum of the global average pooling concerning the $k$ th feature map given by:

$$
\begin{aligned}
S_{c} & =\Sigma_{k} w_{k}^{c} F^{k} \\
& =\Sigma_{k} w_{k}^{c} \Sigma_{x, y} f_{k}(x, y) \\
& =\Sigma_{x, y} \Sigma_{k} w_{k}^{c} f_{k}(x, y)
\end{aligned}
$$

where $f_{k}(x, y)$ denotes the spatial element $(x, y)$ activation in the $k$ th feature map. The class activation map of class $c, M_{c} \in \mathbb{R}^{u \times v}$, is defined as a weighted sum of activation at spatial element $(x, y)$ from all feature maps.

$$
M_{c}(x, y)=\Sigma_{k} w_{k}^{c} f_{k}(x, y)
$$

(Eq. 5)

The definition of the Grad-CAM for a class $c$ is the weighted sum of all feature maps resulting from the last convolution layer in a DCNN. We applied the ReLU function (Eq. 3) to remove the potential influence of negative weights on the class of interest, considering that the spatial elements in the feature maps associated with the negative weights were likely to belong to other categories in the image.

$$
\operatorname{Grad}_{-} M_{c}(x, y)=\operatorname{ReLU}\left(\Sigma_{k} \alpha_{k}^{c} f_{k}(x, y)\right)
$$

where $\alpha_{k}^{c}$ is the weight obtained by computing the gradient of a prediction score and is given by:

$$
\alpha_{k}^{c}=\Sigma_{x, y} \frac{\partial S_{c}}{\partial f_{k}(x, y)}
$$

Figure 1C shows the DCNN model (ResNet-34) block diagram for diagnosis/prognosis, which consists of a three-step pipeline for 1) detecting random CXR images, where the first layers detect edges and shapes while the last layers work on the details; 2) identifying all suspicious patchy infiltrates in the CXR image of the lung; and 3) classifying (i.e., diagnosing) of COVID-19, other viral pneumonias, and normal controls.

The performance of the ResNet-34 was monitored graphically, including the training loss, validation loss, and error rate along epochs. We assessed the accuracy of classification (i.e., diagnosis) by the receiver operating characteristic (ROC) curve, the area under the curve (AUC), sensitivity, and specificity analysis, 


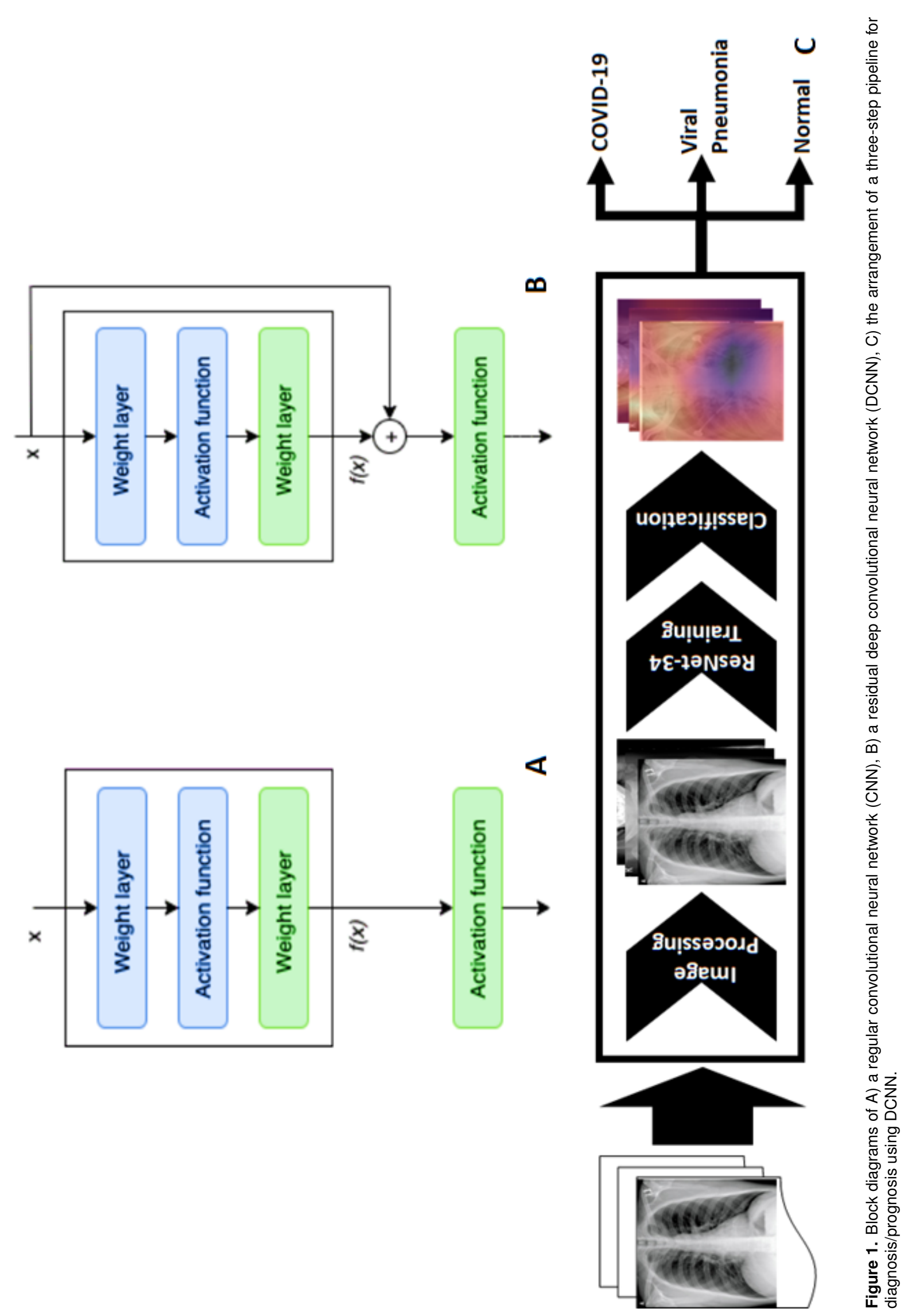



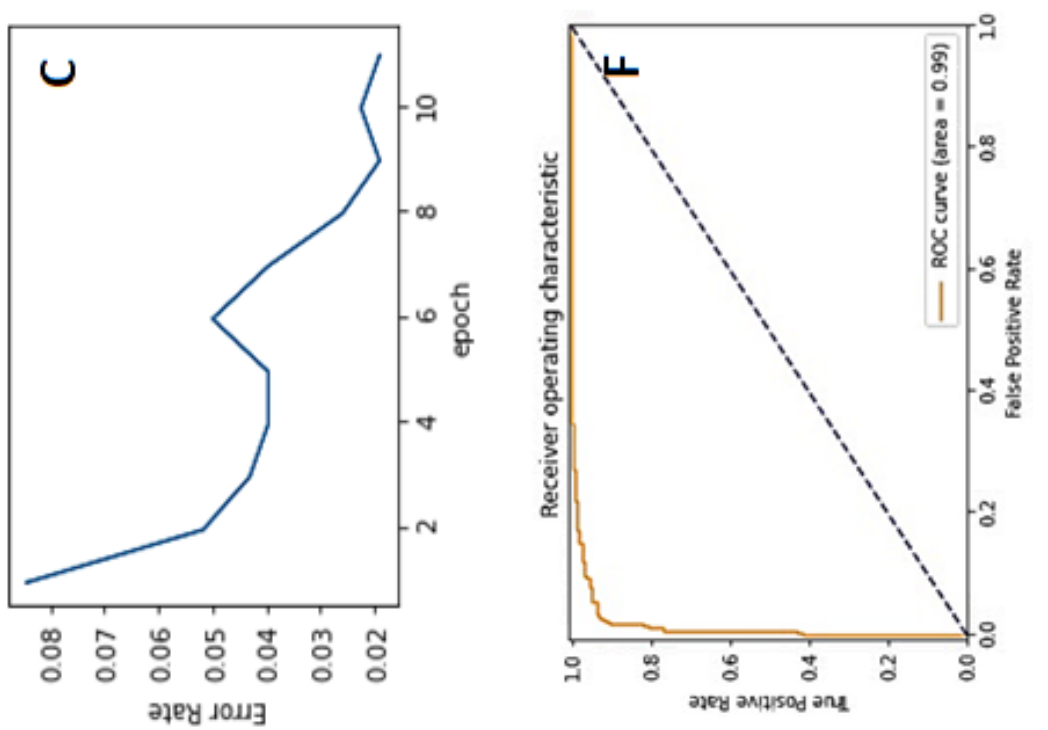


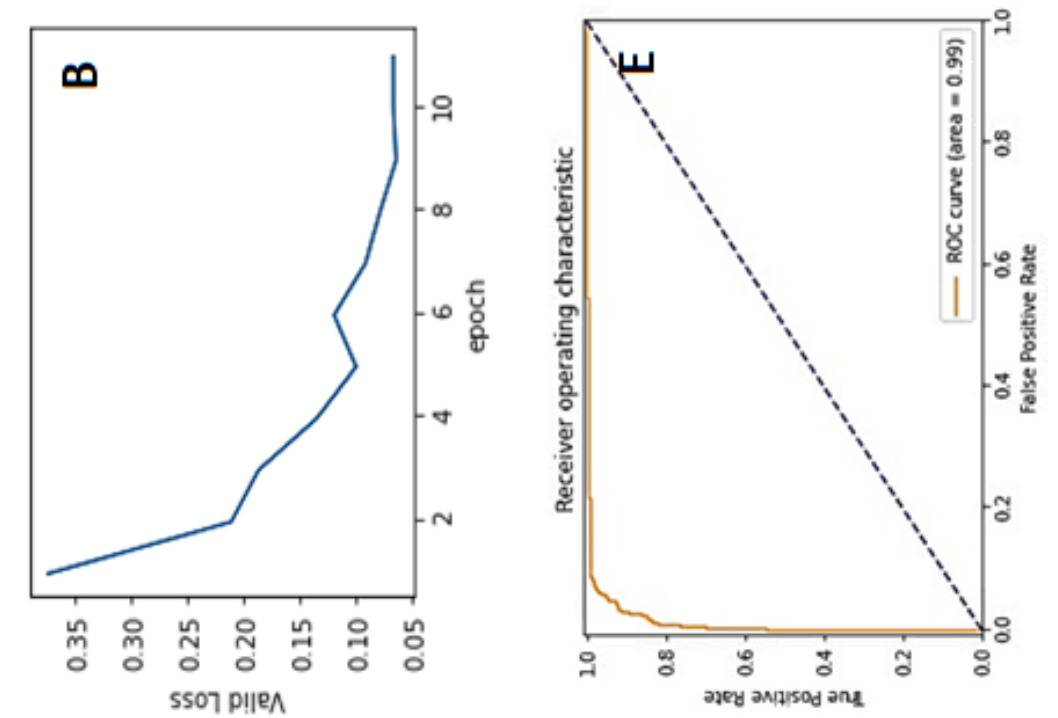

(1)
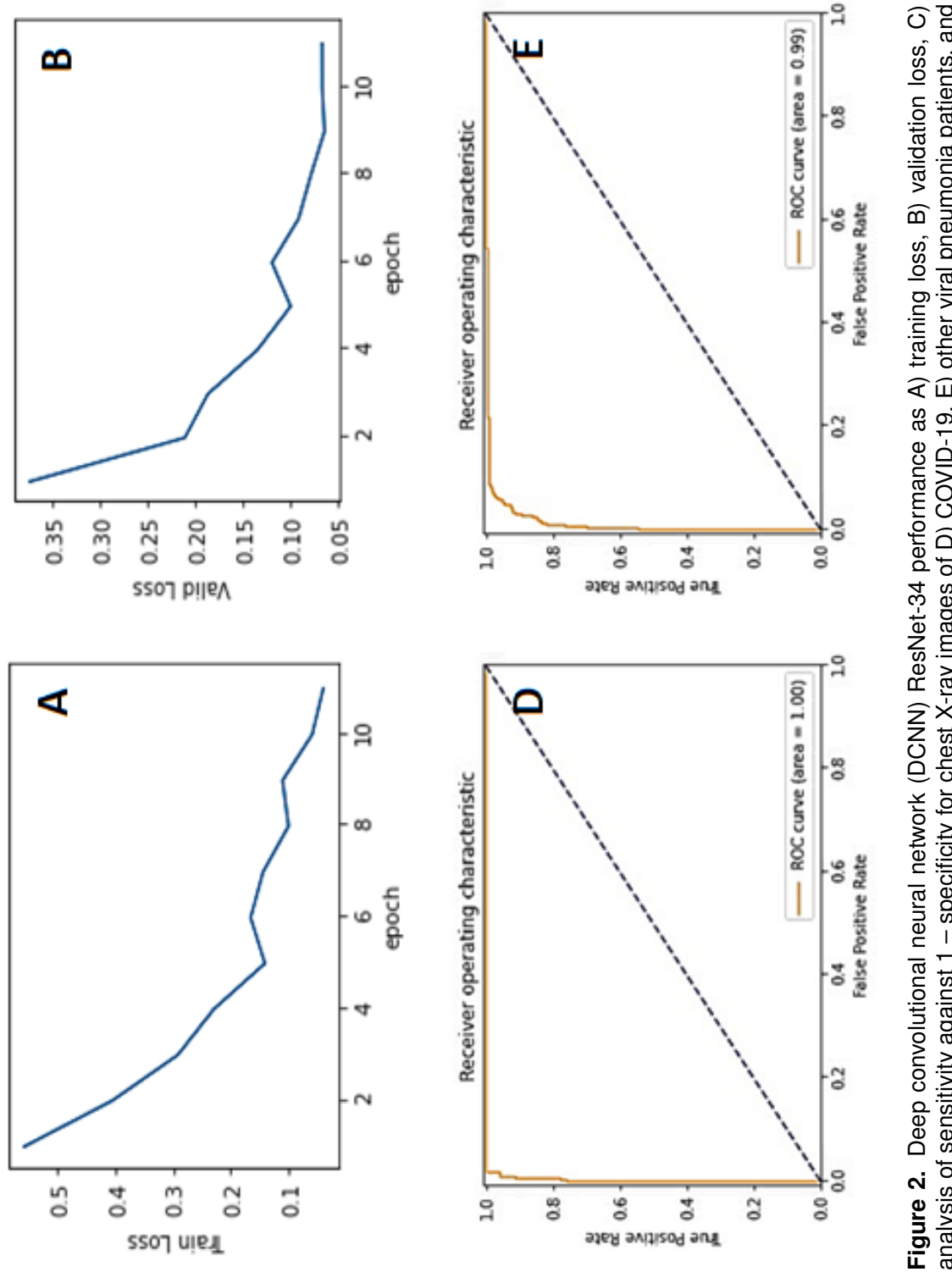
Table 2. Accuracy and error rate of k-fold cross-validation tests.

\begin{tabular}{lcc}
\hline K-fold & Accuracy $(\%)$ & Error rate \\
\hline 1 & 99.14 & $8.61 \times 10^{-3}$ \\
2 & 99.31 & $6.89 \times 10^{-3}$ \\
3 & 99.48 & $5.16 \times 10^{-3}$ \\
4 & 98.62 & $13.77 \times 10^{-3}$ \\
5 & 98.97 & $10.33 \times 10^{-3}$ \\
Average result & 99.11 & $8.95 \times 10^{-3}$ \\
\hline
\end{tabular}

a)

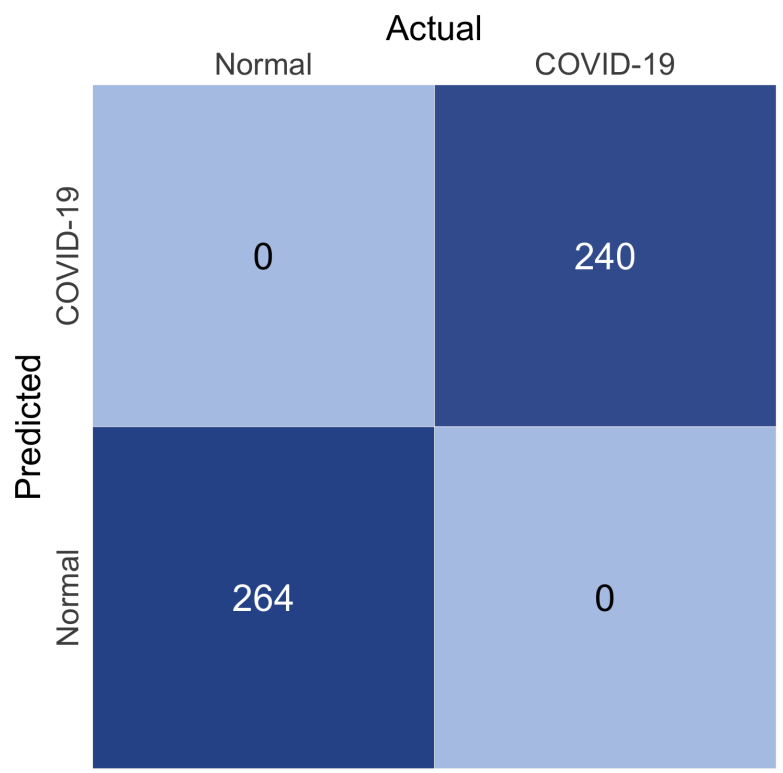

b)

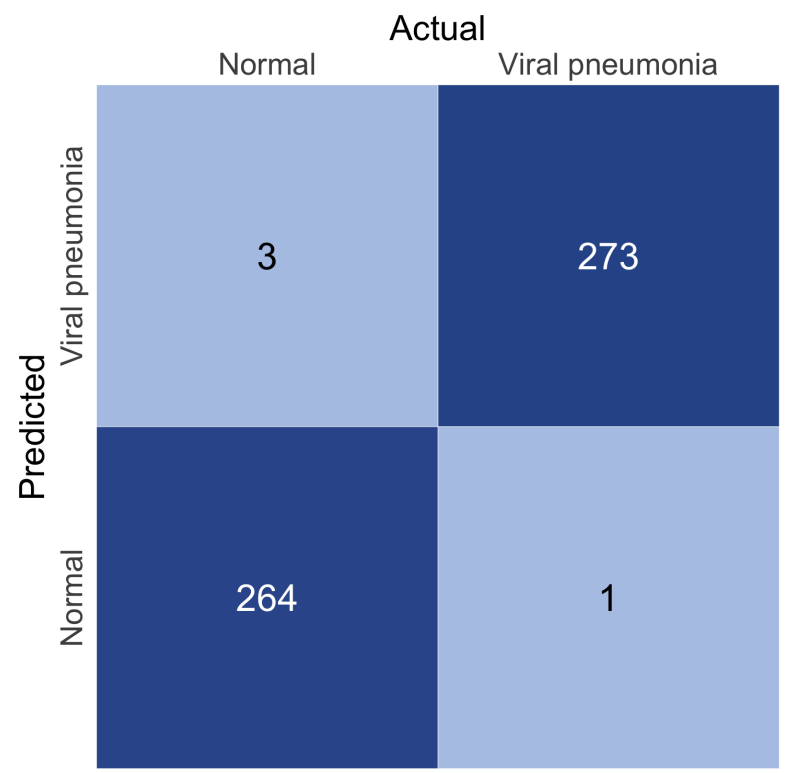

Figure 3. Chi square of ResNet-34 classification results (diagnosis/prognosis) of a) COVID-19 versus normal control chest X-ray images and b) other viral pneumonia versus normal control chest $\mathrm{X}$-ray images.

as well as a chi square for actual versus predicted values of COVID-19 versus normal control CXR images, and another for other viral pneumonia, versus normal control CXR images. We showed samples of grayscale $X$-ray input images and output colored activation map grids for COVID-19, other viral pneumonias, and normal controls. Finally, we carried out k-fold cross-validation tests to classify all 781 source CXR images from COVID-19 $(n=240)$, other viral pneumonias $(n=274)$, and normal controls $(n=267)$, divided equally randomly among each one- to five-fold, respectively.

\section{Results}

The performance of the ResNet-34 model, where the training and validation loss of random subsets of CXR images and the error rate with the number of epochs converge at the end of the training phase. (Figures 2AC) An epoch is a measure of the cycles through the full training process using image batches once to update the ResNet-34 weights. We found that ten epochs were sufficient to avoid both under- and over-fitting, reflecting the high precision of testing in the subset of random CXR images used for the diagnosis/prognosis. Fundamental measures of the diagnostic accuracy of the ResNet-34 model, as of ROC curves of the true positive rate versus the false positive rate for COVID-19, other viral pneumonia, and normal control CXR images, are shown in Figures 2D-F. AUC values for COVID-19, other viral pneumonias, and normal controls were 1.00, 0.99 , and 0.99 , which signify a perfect accuracy in the diagnosis and identical sensitivity and specificity values of 100, 99, and 99\% for the three groups, respectively, by three-fold cross-validation test, as shown in Table 2. In general, the ResNet-34 achieved a high average accuracy of $99.11 \%$ and a low error rate of $8.95 \times$ $10^{-3}$ in classifying all source CXR images.

Moreover, the chi squares of Figure 3 show that the ResNet-34 had identical sensitivity and specificity of 100, 99.6, and $98.9 \%$ in classifying all source CXR im- 
ages of COVID-19, other viral pneumonias, and normal controls, respectively. The overall accuracy of the ResNet-34 for testing the subset of random CXR images used for the diagnosis / prognosis was as high as $99.5 \%$, indicating an error rate as low as $0.5 \%$.

Figures $4 \mathrm{~A}-\mathrm{C}$ show an example of the input CXR grayscale images batch for COVID-19, other viral pneumonias, and normal controls. Figures 4D-F also show an example of the colored activation map of the ResNet-34 final output layer for the three different subsets. The activation map is a proper visual diagnostic representation, highlighting the morphological anomalies of a CXR image that contribute most to the ResNet34 classification process.

\section{Discussion}

X-ray imaging for computer-aided medical detection, classification, and diagnosis of many diseases is an active field of research. It is accessible and can be performed at a low cost using portable units in clinics or homes.[27, 28] Digital image processing effectively visualizes and extracts essential details, with many available techniques for enhancing the quality of a standard X-ray image before the diagnosis. Elsharkawy et al. have recently proposed a pre-processing technique for correcting CXR images for non-uniformity of illumination and delimiting the boundaries of lungs using an automated segmentation routine or manual delineation by radiologists.[28] The technique uses estimates of the Markov-Gibbs random field energy at several radii to compute the cumulative distribution function describing the lung nodules and determine the severity of infection using a neural-network fusion and diagnostic system. Vieira et al. have also proposed image resizing using the maximum window function, which preserves anatomical structures of the chest, to increase the classification rate regardless of the deep learning architectures used.[29]

Radiologists rely on the appearance of CXR images to identify any atypical unilateral patchy infiltration in the lungs of COVID-19 patients, although there no irregularities in the first grades of infection, and, if present, they may be confused with pneumonia.[7] Thus, to train a high-performance DCNN for diagnosis/prognosis of COVID-19 infection, using large datasets for training is essential. However, large repositories containing enough CXR images are not always available. Thus, transfer learning is the base for initializing a DCNN model, fine-tuned using the available limited medical datasets with results that outperform fully trained models under particular conditions.[20] The ResNet-34 model showed a very high performance, as of the convergence of the training loss, validation loss, and the error rate along with the training phase with the number of epochs to zero
(Figures 2A-C). The distinctive nature of ResNet-34, which builds on constructs from pyramidal cells in the cerebral cortex using skip connections or shortcuts to jump over some layers, permits the advantageous reduction of over-fitting and leads to faster optimization of and better overall performance of the model. That is, only ten epochs were required to attain a high precision of testing and cross-validation of the subset of random CXR images without under- or over-fitting (Table 2).

AUC analysis of ROC curves (Figure 2D-F) of truepositive rate (i.e., sensitivity) versus false-positive rate (i.e., 1-specificity) shows that points in the upper left corner signify sensitivity and specificity as high as 100, 99, and 99\% for COVID-19, other viral pneumonias, and normal controls, respectively, by three-fold cross-validation. Generally, ROC curves lie between the lines connecting $(0,0)$ to $(0,1)$ and $(0,1)$ to $(1,1)$ extremes, where the area under the ROC curve is a summary measure that essentially averages the diagnostic accuracy of model predictions. The RSNA and other COVID-19 open access CXR image repositories have formed the basis for many studies for detecting pneumonia and COVID-19.[7, 24] One of these is the recent study by Zhang et al.[30] They have successfully employed a pre-trained 18-layer ResNet using ImageNet weights for screening CXR images to detect, identify, and classify 106 COVID-19 patients and 107 normal controls without any fine-tuning. They showed that the ResNet achieved an AUC of $83.61 \%$ and sensitivity of $71.70 \%$, which they maintain is comparable to the radiologists' performance reported in the literature.

The ResNet-34 had identical sensitivity and specificity of $100,99.6$, and $98.9 \%$ in classifying all source CXR images, without any image augmentation, of COVID-19, other viral pneumonias, and normal controls, respectively (Figure 3). The overall accuracy of the ResNet-34 for testing the subset of random CXR images used for diagnosis/prognosis was as high as $99.5 \%$. The error rate of $0.5 \%$ in the testing phase is much lower than the 3.6\% showed for the benchmarked ResNet-34.[21] Our group has lately shown similar high-performance results for estimating bone mineral density from DXADICOM images with an accuracy of $100 \%$ for the training, cross-validation, and testing phases of an ANN, yet with much-elaborated image processing for feature extraction using both histogram and binary algorithms.[27] The highest accuracy yielded by the automated framework recently proposed by Akram et al. for the rapid diagnosis of COVID-19 from a limited number of CT images of patients from a public domain website, with augmented feature classification using the Naive Bayes classifier, was only $92.6 \%$.[31]

Wang and Wong have recently introduced COVIDNet, an open-source DCNN design tailored for detecting COVID-19, pneumonia, and normal controls from CXR images, which is available to the public.[32] To 

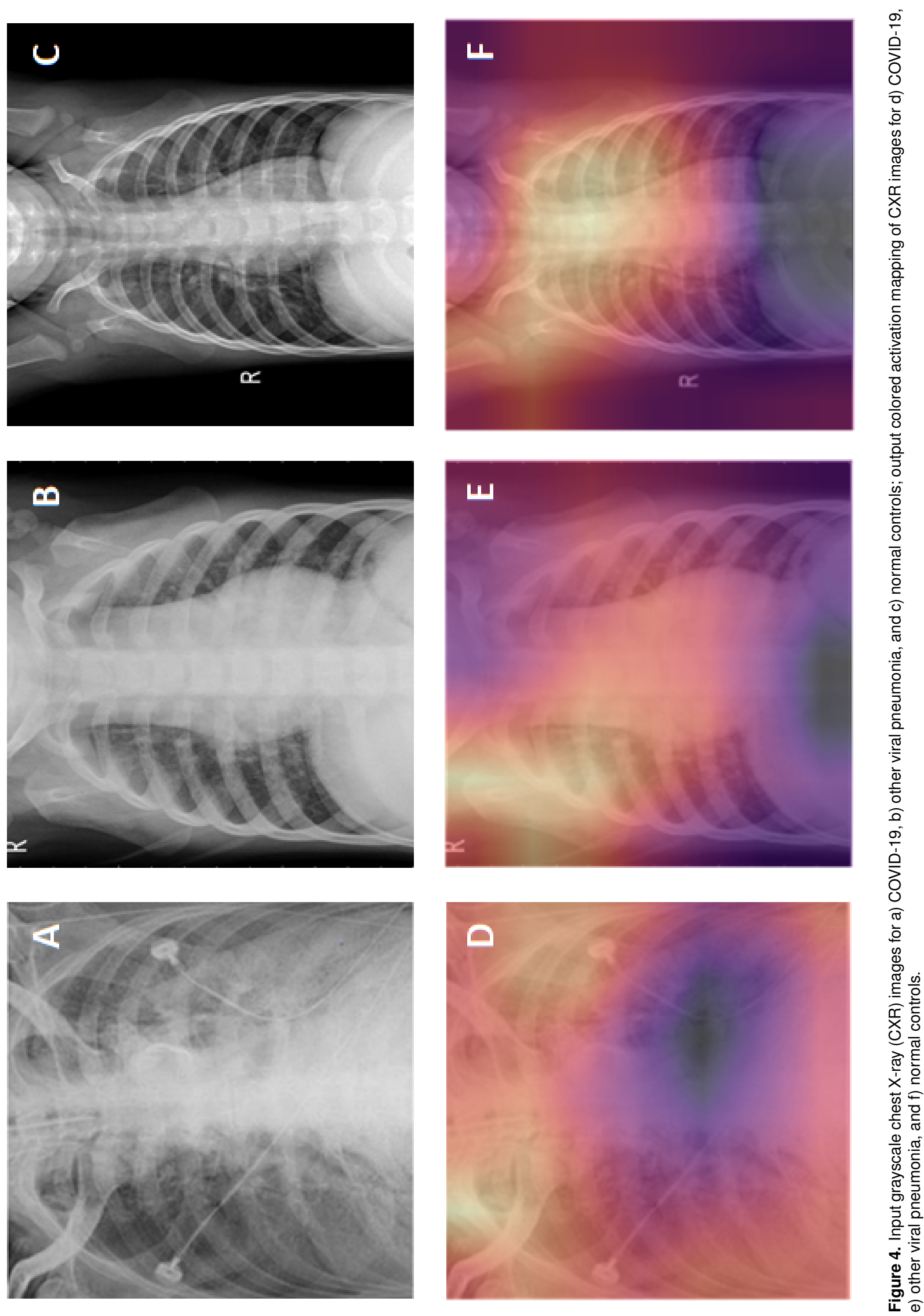
assist radiologists to automatically diagnose COVID19 in X-ray images, Hemdan et al. trained and validated their COVIDX-Net using a small dataset, which included only 25 COVID-19-positive patients.[33] The COVIDX-Net included seven different architectures of DCNN models, such as the modified Visual Geometry Group Network (VGG19) and the second version of Google MobileNet, which classified the patients' normalized intensities of X-ray images as negative or positive COVID-19. The VGG19 and Dense Convolutional Network (DenseNet) models showed a good and similar performance of automated COVID-19 classification with f1-scores of 0.89 and 0.91 for normal controls and COVID-19, respectively. The sensitivity of the COVIDNet was 91, 94, and 95\% for COVID-19, pneumonia patients, and normal controls, respectively, with an overall performance accuracy of $93.3 \%$.

Distinguishing morphological anomalies in CXR images of COVID-19 patients from typical pneumonia patients is not easy. We employed the Grad-CAM method to localize and visualize an ROI within CXR images, which consistently highlights a specific area common in shape, pattern, or location among CXR images within the same class but distinct or different from images in other modality classes.[20] The activation map (Figures 4D-F) is thus a proper visual diagnostic representation, highlighting the morphological anomalies of a CXR image that contribute the most to the ResNet-34 classification process. Li et al. have recently addressed this problem of automatically distinguishing between COVID-19 and typical pneumonia using a U-Net pre-processor network for lung field segmentation, followed by a 3D ResNet-50 using transferred ImageNet weights.[34] Their model attained an accuracy of $87 \%$ using a dataset collected from 6 hospitals. Furthermore, the DenseNet-169 CNN model, tested to identify COVID-19 from CT slices without

Received: June 18, 2021

Accepted: December 9, 2021

Published: December 15, 2021

Copyright: (C) 2021 The author(s). This original article is brought to you for free and open access by ThinkIR: The University of Louisville's Institutional Repository. For more information, please contact thinkir@louisville.edu. This article is and with segmentation, showed $79.5 \%$ and $83.3 \%$ accuracy, respectively.[35] A model based on a combination of U-Net and 3D CNN has identified the presence of COVID-19 with a 90\% accuracy using CT images.[36] An accuracy of $94 \%$ has also been achieved with a ResNet-50-based CNN using transfer learning weighted from the ImageNet.[37]

In conclusion, distinguishing morphological anomalies in CXR images (i.e., opacities and lung consolidations displayed as atypical unilateral patchy infiltration in the lungs) of COVID-19 patients from those of other pneumonia patients is cumbersome. It usually requires a highly expert radiologist and typically ends with ordering other expensive CT scans, which burdens the national health systems of all countries and delays the diagnosis of COVID-19 patients. In the present study, we employed the ResNet-34 for the detection and classification (diagnosis/prognosis) of CXR images of COVID-19, other viral pneumonias, and normal controls from four international sub-databases: the Società Italiana di Radiologia Medica e Interventistica (SIRM), the GitHub database, the Radiology Society of North America (RSNA), and the Kaggle chest X-ray database. Images were resized, normalized without any image augmentation, and arranged in $m$ batches of 16 images before supervised training and validation of the DCNN classifier. The ResNet-34 model achieved identical sensitivity and specificity of $100 \%, 99.6 \%$, and 98.9\% in classifying CXR images of COVID-19, other viral pneumonias, and normal controls, respectively, with an overall accuracy of $99.5 \%$ for the testing subset for diagnosis/prognosis. Based on this high classification precision, we believe that the output activation map of the final layer of the ResNet-34 is a powerful tool for the accurate diagnosis of COVID-19 infection from CXR images.

distributed under the terms of the Creative Commons Attribution 4.0 International License (CC BY 4.0), which permits unrestricted use, distribution, and reproduction in any medium, provided the original author and source are credited.

Funding Source: The author(s) received no specific funding for this work.

Conflict of Interest: All authors declared no conflict of interest in relation to the main objective of this work. 


\section{References}

1. World Health Organization. WHO Coronavirus (COVID19) Dashboard. Available at: https://covid19.who.int/ Accessed 14 October 2021.

2. Zhai $P$, Ding $Y$, Wu $X$, Long J, Zhong $Y, L i \quad Y$. The epidemiology, diagnosis and treatment of COVID19. Int J Antimicrob Agents 2020; 55(5):105955. doi: 10.1016/j.jjantimicag.2020.105955. PMID: 32234468.

3. Russakovsky O, Deng J, Su H, et al. ImageNet Large Scale Visual Recognition Challenge. Int J Comput Vis 2015; 115(3):211-52. doi: 10.1007/s11263-015-0816-y.

4. To KK, Tsang OT, Yip CC, et al. Consistent detection of 2019 novel coronavirus in saliva. Clin Infect Dis 2020; 71(15):841-3. doi: 10.1093/cid/ciaa149. PMID: 32047895.

5. Wang $W, X u Y$, Gao R, et al. Detection of SARS-CoV2 in different types of clinical specimens. JAMA 2020; 323(18):1843-4. doi: 10.1001/jama.2020.3786. PMID: 32159775

6. Ai T, Yang Z, Hou H, et al. Correlation of chest CT and RT-PCR testing for coronavirus disease 2019 (COVID-19) in China: A report of 1014 cases. Radiology 2020; 296(2):E32E40. doi: 10.1148/radiol.2020200642. PMID: 32101510.

7. Cohen JP, Morrison P, Dao L, Roth K, Duong TQ, Ghassemi M. COVID-19 image data collection: Prospective predictions are the future. arXiv [Preprint]. 2020 Available at: http://arxiv.org/abs/2006.11988.

8. Kipritidis J, Hofman MS, Siva S, et al. Estimating lung ventilation directly from 4D CT Hounsfield unit values. Med Phys 2016; 43(1):33-43. doi: 10.1118/1.4937599.

9. Fried DV, Tucker SL, Zhou S, et al. Prognostic value and reproducibility of pretreatment $\mathrm{CT}$ texture features in stage III non-small cell lung cancer. Int J Radiat Oncol Biol Phys 2014; 90(4):834-42. doi: 10.1016/j.ijrobp.2014.07.020. PMID: 25220716.

10. Wu T, Sultan LR, Tian J, Cary TW, Sehgal CM. Machine learning for diagnostic ultrasound of triple-negative breast cancer. Breast Cancer Res Treat 2019; 173(2):365-73. doi: 10.1007/s10549-018-4984-7. PMID: 30343454.

11. Uthoff J, Stephens MJ, Newell JD, Jr., et al. Machine learning approach for distinguishing malignant and benign lung nodules utilizing standardized perinodular parenchymal features from CT. Med Phys 2019; 46(7):3207-16. doi: 10.1002/mp.13592. PMID: 31087332.

12. Yang $X$, Jeong JJ, Lei $Y$, et al. Machine-learning-based classification of glioblastoma using MRI-based radiomic features. In: Computer-Aided Diagnosis: SPIE, 2019:152.

13. Wong KC, Moradi M, Wu J, Syeda-Mahmood T. Identifying disease-free chest X-ray images with deep transfer learning: SPIE, 2019.

14. Gunčar $G$, Kukar $M$, Notar $M$, et al. An application of machine learning to haematological diagnosis. Sci Rep 2018; 8(1):411. doi: 10.1038/s41598-017-18564-8. PMID: 29323142
15. Arai $Y$, Kondo $T$, Fuse $K$, et al. Using a machine learning algorithm to predict acute graft-versus-host disease following allogeneic transplantation. Blood Adv 2019; 3(22):3626-34. doi: 10.1182/bloodadvances.2019000934. PMID: 31751471.

16. Diamond LW, Mishka VG, Seal AH, Nguyen DT. Multiparameter interpretative reporting in diagnostic laboratory hematology. Int J Biomed Comput 1994; 37(3):211-24. doi: 10.1016/0020-7101(94)90120-1. PMID: 7705904.

17. Nguyen DT, Diamond LW, Cavenagh JD, Parameswaran $\mathrm{R}$, Amess JA. Haematological validation of a computerbased bone marrow reporting system. J Clin Pathol 1997; 50(5):375-8. doi: 10.1136/jcp.50.5.375. PMID: 9215118.

18. D'Onofrio G, Zini G. Morphology of the blood. Oxford: Butterworth-Heinemann, 1998.

19. Kantardzic $M$, Djulbegovic $B$, Hamdan $H$. A data-mining approach to improving Polycythemia Vera diagnosis. Comput Ind Eng 2002; 43(4):765-73. doi: 10.1016/S03608352(02)00138-9.

20. Kim I, Rajaraman S, Antani S. Visual interpretation of convolutional neural network predictions in classifying medical image modalities. Diagnostics (Basel) 2019; 9(2). doi: 10.3390/diagnostics9020038. PMID: 30987172.

21. Koustubh. ResNet, AlexNet, VGGNet, Inception: Understanding various architectures of convulational networks. Available at: https://cv-tricks.com/cnn/understand-resnetalexnet-vgg-inception/. Accessed 26 February 2021.

22. Società Italiana di Radiologia Medica e Interventistica. COVID-19 database - SIRM. Available at: https://www.sirm. org/category/senza-categoria/covid-19/. Accessed 26 February 2021.

23. Cohen JP, Morrison $P$, Dao L, Roth K, Duong TQ, Ghassem M. COVID-19 image data collection. Available at: https:// github.com/ieee8023/covid-chestxray-dataset. Accessed 26 February 2021.

24. Summers R. RSNA pneumonia detection challenge. Available at: https://nihcc.app.box.com/v/ChestXray-NIHCC/ folder/36938765345. Accessed 26 February 2021.

25. Kermany DS, Goldbaum M, Cai W, et al. Identifying medical diagnoses and treatable diseases by imagebased deep learning. Cell 2018; 172(5):1122-31.e9. doi: 10.1016/j.cell.2018.02.010. PMID: 29474911.

26. Paszke A, Gross S, Massa F, et al. PyTorch: An imperative style, high-performance deep learning library. In: 33rd Conference on Neural Information Processing Systems. Vancouver, Canada: Curran Associates, Inc., 2019.

27. Mohamed El, Meshref RA, Abdel-Mageed SM, Moustafa $\mathrm{MH}$, Badawi MI, Darwish SH. A novel morphological analysis of DXA-DICOM images by artificial neural networks for estimating bone mineral density in health and disease. J Clin Densitom 2019; 22(3):382-90. doi: 10.1016/j.jocd.2018.08.006. PMID: 30292570. 
28. Elsharkawy M, Sharafeldeen A, Taher F, et al. Early assessment of lung function in coronavirus patients using invariant markers from chest X-rays images. Sci Rep 2021; 11(1):12095. doi: 10.1038/s41598-021-91305-0. PMID: 34103587.

29. Vieira $P$, Sousa $O$, Magalhães $D$, Rabêlo $R$, Silva $R$. Detecting pulmonary diseases using deep features in $X$ ray images. Pattern Recognit 2021; 119:108081. doi: 10.1016/j.patcog.2021.108081. PMID: 34149099.

30. Zhang J, Xie Y, Pang G, et al. Viral pneumonia screening on chest $X$-ray images using confidence-aware anomaly detection. arXiv [Preprint]. 2020 Available at: http://arxiv.org/ abs/2003.12338.

31. Akram $T$, Attique $M$, Gul $S$, et al. A novel framework for rapid diagnosis of COVID-19 on computed tomography scans. Pattern Anal Appl 2021:1-14. doi: 10.1007/s10044020-00950-0. PMID: 33500681.

32. Wang L, Wong A. Covid-net: A tailored deep convolutional neural network design for detection of COVID-19 cases from chest X-ray images. arXiv [Preprint]. 2020 Available at: http://arxiv.org/abs/2003.09871.
33. Hemdan EE-D, Shouman MA, Karar ME. COVIDX-Net: A framework of deep learning classifiers to diagnose COVID19 in X-ray images. arXiv [Preprint]. 2020 Available at: http://arxiv.org/abs/2003.11055.

34. Li L, Qin L, Xu Z, et al. Using artificial intelligence to detect COVID-19 and community-acquired pneumonia based on pulmonary CT: Evaluation of the diagnostic accuracy. Radiology 2020; 296(2):E65-e71. doi: 10.1148/radiol.2020200905. PMID: 32191588.

35. Yang X, He X, Zhao J, Zhang Y, Zhang S, Xie P. COVIDCT-Dataset: A CT scan dataset about COVID-19. arXiv [Preprint]. 2020 Available at: http://arxiv.org/abs/2003.13865.

36. Zheng $\mathrm{C}$, Deng $\mathrm{X}, \mathrm{Fu} \mathrm{Q}$, et al. Deep learningbased detection for COVID-19 from chest CT using weak label. medRxiv [Preprint]. 2020 doi: 10.1101/2020.03.12.20027185.

37. Gozes O, Frid-Adar M, Greenspan H, et al. Rapid Al development cycle for the coronavirus (COVID-19) pandemic: Initial results for automated detection \& patient monitoring using deep learning CT image analysis. arXiv [Preprint]. 2020 Available at: http://arxiv.org/abs/2003.05037. 